\title{
Management of a Dog with Poorly Regulated Diabetes Mellitus, Chronic Pancreatitis, and Suspected Atopy with Cyclosporine
}

\author{
Jörg M. Steiner ${ }^{1}$ and Brian J. Huber ${ }^{2}$ \\ ${ }^{1}$ Gastrointestinal Laboratory, Department of Small Animal Clinical Sciences, College of Veterinary Medicine and Biomedical Sciences, \\ Texas A\&M University, 4474 TAMU, College Station, TX 77843-4474, USA \\ ${ }^{2}$ Animal Hospital of Rowlett \& Diagnostic Center, Rowlett, TX 75088, USA
}

Correspondence should be addressed to Jörg M. Steiner, jsteiner@cvm.tamu.edu

Received 21 December 2011; Accepted 24 February 2012

Academic Editors: P. Roccabianca and A. Sainz

Copyright (C) 2012 J. M. Steiner and B. J. Huber. This is an open access article distributed under the Creative Commons Attribution License, which permits unrestricted use, distribution, and reproduction in any medium, provided the original work is properly cited.

A 3-year-and-9-months old male neutered Bichon Frise was presented for a second opinion for diabetes mellitus, weight loss, pruritus, and loss of hair. During further work-up, the dog was diagnosed with uncontrolled diabetes mellitus and concurrent diagnoses of pancreatitis and atopy were also suspected. Multiple adjustments of insulin therapy did not improve control of diabetes mellitus. Also, a variety of different treatments failed to improve pruritus. The dog was seen by a veterinary dermatologist who further suspected atopy and started treatment with cyclosporine. Pruritus improved and coincidentally serum Spec cPL and fructosamine concentrations normalized after therapy, suggesting the possibility that cyclosporine may have controlled pancreatic inflammation and improved control of diabetes mellitus. This case report would suggest that further research into autoimmunity in dogs with chronic pancreatitis is warranted. Also, a controlled study is needed and in progress before the use of cyclosporine in dogs with chronic pancreatitis or a subgroup thereof can be advocated.

\section{Introduction}

Chronic pancreatitis in dogs can lead to progressive destruction of the pancreas, potentially leading to diabetes mellitus and/or exocrine pancreatic insufficiency [1]. Thus, some patients may experience concurrent pancreatic inflammation and diabetes mellitus. It has been recognized that these patients can be frustrating to regulate and chronic pancreatitis should be considered as a possible cause for unregulated diabetes mellitus in the dog [2].

Over the last two decades, autoimmune pancreatitis (AIP) has been described as a cause of pancreatitis in humans $[3,4]$. The presentation of humans with AIP can vary, but there are several key features: lymphocytic-plasmacytic inflammation that is centred around pancreatic ducts, pancreatic mass formation, IgG4-positive lymphocytes, and response to corticosteroid therapy [5]. It has also been postulated that some cases of chronic pancreatitis in dogs may also be immune mediated, and anecdotally, several dogs with chronic pancreatitis have responded to corticosteroid therapy $[1,6]$. Also, dogs with chronic pancreatitis usually have lymphocytic-plasmacytic infiltration $[7,8]$. However, this inflammatory infiltration is not centred around pancreatic ducts and is usually not associated with the formation of mass lesions (unpublished, data 2009). However, it should be recognized that AIP, though it may exist in dogs, may show significant differences to this disease in humans.

Cyclosporine is an immunosuppressive agent that acts by suppression of interleukin 2 (IL-2) synthesis from helper T lymphocytes and IL-1 from monocytes [9]. Cyclosporine was initially used for the prevention of organ rejection in transplant patients, but over the last two decades cyclosporine has also been used for the treatment of a variety of autoimmune diseases in humans, such as rheumatoid arthritis, psoriasis, and atopic dermatitis $[10,11]$. Cyclosporine has also been used in dogs, mainly for the treatment of atopy, but recently also for idiopathic inflammatory bowel disease $[12,13]$. A newer formulation of cyclosporine (Atopica, Novartis) is considered safer than original formulations that required careful monitoring of serum levels of cyclosporine in order 
to prevent side effects due to overdosing or lack of action due to underdosing.

\section{Case Report}

A 3-year-and-9-month old male neutered Bichon Frise was presented for a second opinion for diabetes mellitus, weight loss, pruritus, and loss of hair. The previous history included chronic skin problems for about 4 months duration and being diagnosed with diabetes mellitus approximately one month earlier. Upon initial presentation, the dog weighed $9.0 \mathrm{~kg}$ and had normal vital signs. The dog was fed a low-fat diet (Prescription diet w/d canine dry, Hill's Pet Nutrition) and also received carrots and green beans as a treat. The dog was being treated with 4 units of porcine insulin zinc suspension (Vetsulin, Intervet) q $12 \mathrm{hrs} \mathrm{SC}$ and $25 \mathrm{mg}$ hydroxyzine q $12 \mathrm{hrs}$ PO. Physical examination revealed plaque formation and minimal gingivitis, but no other abnormalities were noted. The only abnormalities on a complete blood count included moderate eosinophilia with 3,770 eosinophils/ $\mu \mathrm{L}$ and a slight thrombocytosis of 474,000 platelets $/ \mu \mathrm{L}$. The serum was moderately lipemic and was spun down to decrease lipemia. The only abnormalities on a serum chemistry profile were an increased alkaline phosphatase activity of $611 \mathrm{U} / \mathrm{L}$ (reference range: 5-131 $\mathrm{U} / \mathrm{L}$ ), an increased cholesterol concentration of $10.6 \mathrm{mmol} / \mathrm{L}$ (reference range: $2.4-8.4 \mathrm{mmol} / \mathrm{L}$ ), and an increased glucose concentration of $20.9 \mathrm{mmol} / \mathrm{L}$ (reference range: 3.9 $7.7 \mathrm{mmol} / \mathrm{L}$ ). Serum triglyceride concentration was not part of the routine chemistry profile and was not assessed. Serum Spec cPL (IDEXX Laboratories) concentration was $>1000 \mu \mathrm{g} / \mathrm{L}$, suggestive of pancreatitis. Urinalysis showed a urine specific gravity of 1.047 and a urine glucose of $55.5 \mathrm{mmol} / \mathrm{L}$, but the urine sample was negative for ketone bodies. Urine culture was also negative. At that point uncontrolled diabetes mellitus was confirmed, a diagnosis of atopy was suspected based on clinical signs, and pancreatitis was suspected based on the severely increased serum Spec cPL concentration. The dog was continued to be managed on Vetsulin at $4 \mathrm{U}$ q $12 \mathrm{hrs} \mathrm{SC}$, but was switched to clemastine fumarate at $1.34 \mathrm{mg} \mathrm{q} 12 \mathrm{hrs}$ PO for the suspected atopy. Also, a flea prevention protocol was started at that time. The dog appeared to be more comfortable at home and was reevaluated 2 weeks later. At that point the dog had lost some more weight (body weight $8.8 \mathrm{~kg}$ ). The dog showed mild erythema and was pruritic during the exam. No other abnormalities were observed. Based on the continued skin problems, the dog was switched to another antihistamine and soothing shampoo baths. A serial blood glucose curve showed that the initial glucose surpassed the upper detection limit $(41.6 \mathrm{mmol} / \mathrm{L})$ of the glucometer (AlphaTRAK, Abbott Animal Health). The lowest serum glucose concentration was $13.4 \mathrm{mmol} / \mathrm{L}$ and was reached 4 hours after insulin administration. Serum glucose concentration was back above the detection limit of the glucometer 8 hours after insulin administration. Thus, insulin therapy was again judged to be inadequate. The dog was switched to an ultra-low-fat diet (Digestive Low Fat LF dry, Royal Canin), and the Vetsulin dose was increased to $5 \mathrm{U}$ q $12 \mathrm{hrs}$ SC. Two weeks later another glucose curve was performed, and once again the insulin therapy was judged to be inadequate. Also, the owners reported that the dog was still pruritic. The Vetsulin dose was increased to $6 \mathrm{U} \mathrm{q} 12 \mathrm{hrs} \mathrm{SC}$ and another glucose curve was repeated 2 weeks later. Again, insulin therapy was judged to be inadequate. A serum fructosamine concentration was measured at that time and was found to be $548 \mu \mathrm{mol} / \mathrm{L}$ (laboratory 1: reference range: $260-376 \mu \mathrm{mol} / \mathrm{L}$ ) further supporting insufficient insulin therapy. After consultation with a dermatologist, the dog was started on $50 \mathrm{mg}$ q $24 \mathrm{hrs}$ PO cyclosporine to try to control the suspected atopy. Four weeks after initiation of cyclosporine therapy, the dog was presented for a reevaluation and was judged to be doing well. Pruritus had decreased significantly, and the dog had gained weight (body weight $9.7 \mathrm{~kg}$ ). Serum fructosamine concentration was $473 \mu \mathrm{mol} / \mathrm{L}$ (laboratory 2: reference range: $142-450 \mu \mathrm{mol} / \mathrm{L} ;<500 \mu \mathrm{mol} / \mathrm{L}=$ "good control"). After another month, the dog was still doing well clinically. Serum fructosamine was repeated (laboratory 1: reference range: $260-378 \mu \mathrm{mol} / \mathrm{L}$ ) and was $336 \mu \mathrm{mol} / \mathrm{L}$, which was once again considered as an indication of sufficient glycemic control. Also, serum Spec cPL concentration was repeated and was $<30 \mu \mathrm{g} / \mathrm{L}$ (reference range: $<200 \mu \mathrm{g} / \mathrm{L}$ ). After another month the dog was again reevaluated and was again judged to be doing well with a thicker hair coat than before. He was still on Vetsulin at $6 \mathrm{U}$ SC q $12 \mathrm{hrs}$ and cyclosporine at $50 \mathrm{mg}$ PO q $24 \mathrm{hrs}$. Serum fructosamine concentration was $467.1 \mu \mathrm{mol} / \mathrm{L}$ (laboratory 3: no reference range given, $400-500 \mu \mathrm{mol} / \mathrm{L}=$ "good glycemic control"). Serum Spec cPL concentration was once again $<30 \mu \mathrm{g} / \mathrm{L}$.

\section{Discussion}

The current case is interesting for several reasons. First, this dog had poorly regulated diabetes mellitus and an increased serum Spec cPL concentration, suggesting that chronic pancreatitis may have led to poor glycaemic control in this dog. Secondly, this patient was not specifically treated for pancreatitis other than changing from a low-fat diet to an ultra-low-fat diet, yet serum Spec cPL concentration normalized after initiation of cyclosporine therapy. Finally, the dog had consistently good regulation of his diabetes mellitus and a normal serum Spec cPL concentration after treatment with cyclosporine, suggesting that immunosuppression through the action of cyclosporine may have had an ameliorating effect on pancreatic inflammation, which may have led to a more stable regulation of diabetes mellitus.

There are several limitations to this case report. Most importantly, a tissue sample of the pancreas to definitively diagnose pancreatitis could not be collected in this case. Also, an ultrasound examination was not performed, which would have been helpful to support the suspected diagnosis of pancreatitis. However, it should be noted that recent studies have shown that serum Spec cPL concentration is highly specific for pancreatic origin; thus, the serum Spec cPL concentration of $>1000 \mu \mathrm{g} / \mathrm{L}$ would have unlikely been due to another cause other than pancreatic inflammation [14]. The second limitation of this report is that there is no proof that it was the action of cyclosporine that led 
to the normalization of the serum Spec cPL concentration and the successful regulation of diabetes mellitus. However, it would appear unlikely that the diabetes mellitus in this patient could not be regulated for 4 months, despite multiple glucose curves and adjustments of insulin dose, and then spontaneously improved without any other adjustment in management other than switching the diet. This is especially interesting when considering that the insulin dose was held at $6 \mathrm{Uq} 12 \mathrm{hrs}$ during this time. It is thus reasonable to hypothesize that the positive response in this patient was due to cyclosporine therapy.

It should also be pointed out that the suspected diagnosis of atopy was merely based on the clinical evaluation of the patient and could not be definitively verified. However, whether or not this patient did have atopy is irrelevant for this report and the suspected clinical response of the chronic pancreatitis and the previously poorly controlled diabetes mellitus to the treatment with cyclosporine.

The positive effect of cyclosporine suspected in this patient would suggest that further research into the role of autoimmunity in the pathogenesis of chronic pancreatitis in dogs is warranted. Also, the potential role of cyclosporine in the management of chronic pancreatitis in dogs must be further assessed by controlled studies before routine use can be advocated. Such a study is currently under way at Texas A\&M University.

\section{Abbreviation}

Spec cPL: Canine Specific Pancreatic lipase.

\section{References}

[1] J. M. Steiner, "Exocrine pancreas," in Small Animal Gastroenterology, J. M. Steiner, Ed., pp. 283-306, SchlüterscheVerlagsgesellschaft mbH, Hanover, Germany, 2008.

[2] J. S. Rand, L. M. Fleeman, H. A. Farrow, D. J. Appleton, and R. Lederer, "Canine and feline diabetes mellitus: nature or nurture?” Journal of Nutrition, vol. 134, pp. 2072S-2080S, 2004.

[3] N. I. Church, S. P. Pereira, M. G. Deheragoda et al., "Autoimmune pancreatitis: clinical and radiological features and objective response to steroid therapy in a UK series," American Journal of Gastroenterology, vol. 102, no. 11, pp. 2417-2425, 2007.

[4] D. L. Finkelberg, D. Sahani, V. Deshpande, and W. R. Brugge, "Autoimmune pancreatitis," New England Journal of Medicine, vol. 355, no. 25, pp. 2670-2676, 2006.

[5] S. Detlefsen, A. Mohr Drewes, M. Vyberg, and G. Klöppel, "Diagnosis of autoimmune pancreatitis by core needle biopsy: application of six microscopic criteria," Virchows Archiv, vol. 454, no. 5, pp. 531-539, 2009.

[6] P. J. Watson, J. Archer, A. J. Roulois, T. J. Scase, and M. E. Herrtage, "Observational study of 14 cases of chronic pancreatitis in dogs," Veterinary Record, vol. 167, no. 25, pp. 968-976, 2010.

[7] S. Newman, J. Steiner, K. Woosley, L. Barton, C. Ruaux, and D. Williams, "Localization of pancreatic inflammation and necrosis in dogs," Journal of Veterinary Internal Medicine, vol. 18, no. 4, pp. 488-493, 2004.
[8] P. J. Watson, A. J. A. Roulois, T. Scase, P. E. J. Johnston, H. Thompson, and M. E. Herrtage, "Prevalence and breed distribution of chronic pancreatitis at post-mortem examination in first-opinion dogs," Journal of Small Animal Practice, vol. 48, no. 11, pp. 609-618, 2007.

[9] L. Santori, M. Rastelli, B. Arena, and M. A. Morleo, "Cyclosporine: update on pharmacology, analytical techniques and therapeutic monitoring," Bollettino Chimico Farmaceutico, vol. 136, no. 9, pp. 577-588, 1997.

[10] J. Cather and A. Menter, "Novel therapies for psoriasis," American Journal of Clinical Dermatology, vol. 3, no. 3, pp. 159-173, 2002.

[11] P. Sarzi-Puttini, E. D’Ingianna, M. Fumagalli et al., "An open, randomized comparison study of cyclosporine a, cyclosporine $\mathrm{A}+$ methotrexate and cyclosporine $\mathrm{A}+$ hydroxychloroquine in the treatment of early severe rheumatoid arthritis," Rheumatology International, vol. 25, no. 1, pp. 15-22, 2005.

[12] K. Allenspach, S. Rüfenacht, S. Sauter et al., "Pharmacokinetics and clinical efficacy of cyclosporine treatment of dogs with steroid-refractory inflammatory bowel disease," Journal of Veterinary Internal Medicine, vol. 20, no. 2, pp. 239-244, 2006.

[13] D. C. Robson and G. G. Burton, "Cyclosporin: applications in small animal dermatology," Veterinary Dermatology, vol. 14, no. 1, pp. 1-9, 2003.

[14] S. C. Neilson-Carley, J. E. Robertson, S. J. Newman et al., "Specificity of a canine pancreas-specific lipase assay for diagnosing pancreatitis in dogs without clinical or histologic evidence of the disease," American Journal of Veterinary Research, vol. 72, no. 3, pp. 302-307, 2011. 

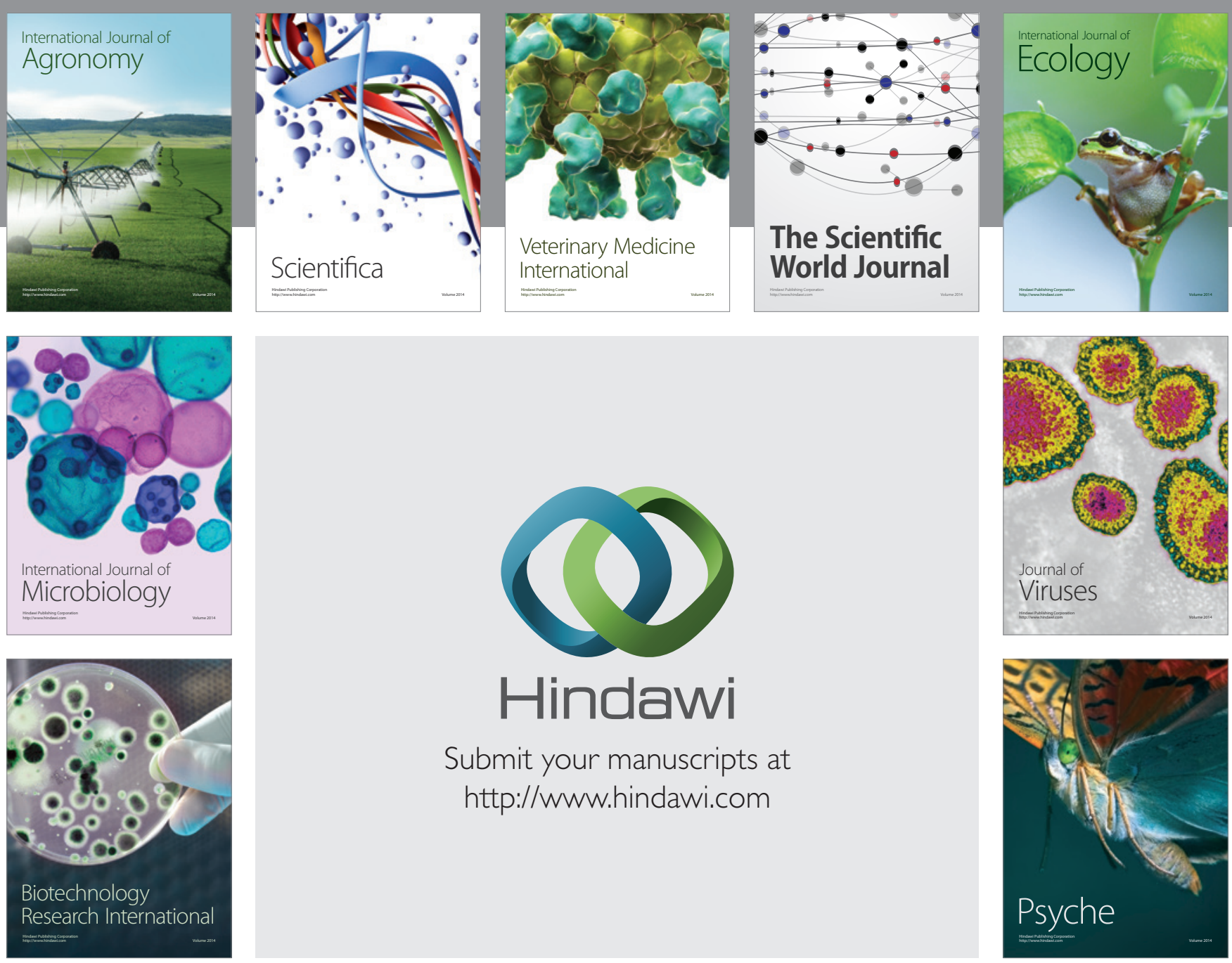

Submit your manuscripts at

http://www.hindawi.com
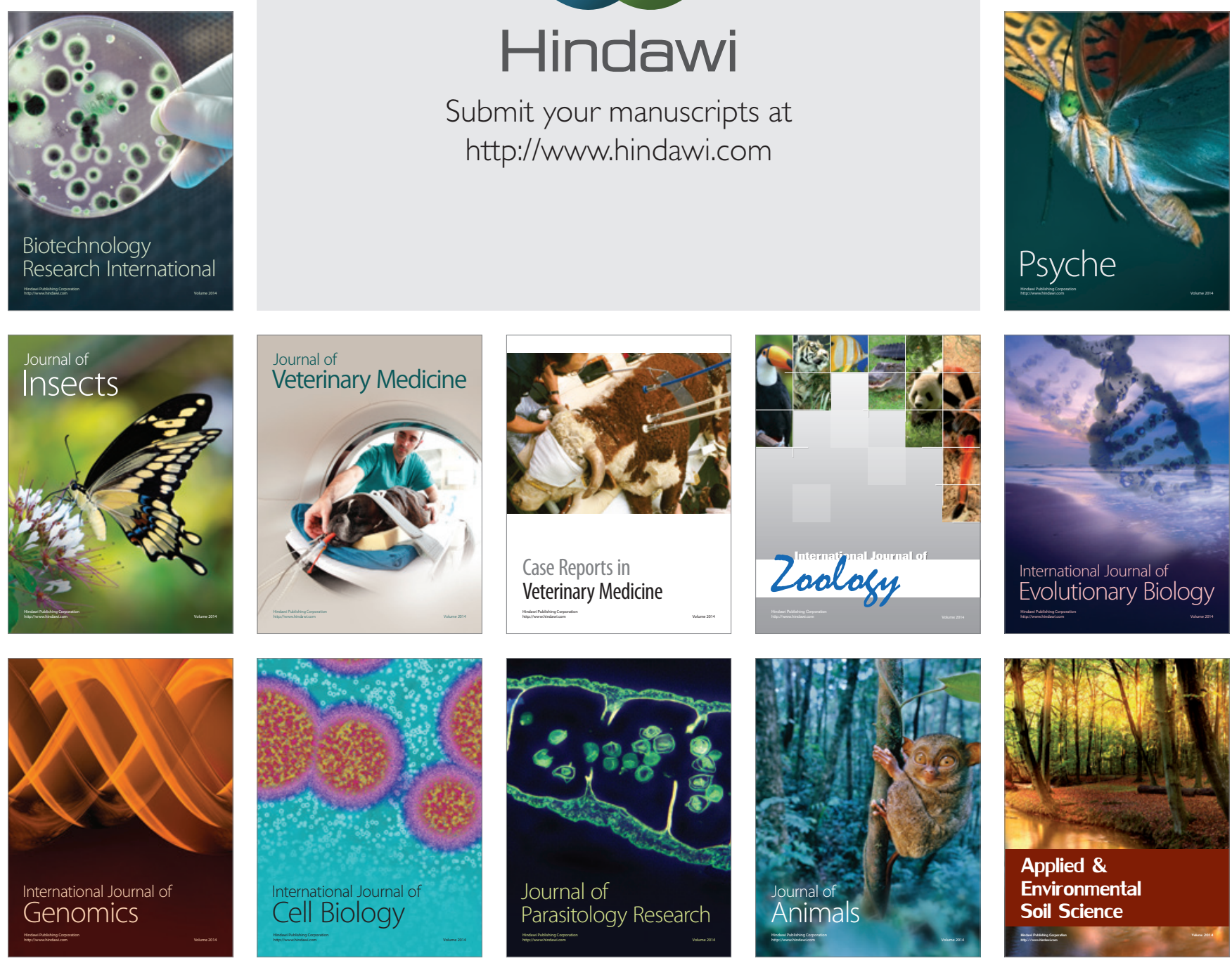